\title{
The linear stability of a Stokes layer with an imposed axial magnetic field
}

\author{
CHRISTIAN THOMAS ${ }^{1}$, ANDREW P. BASSOM ${ }^{1} \dagger$ \\ AND CHRISTOPHER DAVIES ${ }^{2}$ \\ ${ }^{1}$ School of Mathematics \& Statistics, University of Western Australia, Crawley, WA 6009, Australia \\ ${ }^{2}$ School of Mathematics, Cardiff University, Cardiff CF24 4AG, UK
}

(Received 27 June 2010; revised 2 August 2010; accepted 4 August 2010; first published online 27 September 2010)

The effects of a uniform axial magnetic field directed towards an oscillating wall in a semi-infinite viscous fluid (or Stokes layer) is investigated. The linear stability and disturbance characteristics are determined using both Floquet theory and via direct numerical simulations. Neutral stability curves and critical parameters for instability are presented for a range of magnetic field strengths. Results indicate that a magnetic field directed towards the boundary wall is stabilizing, which is consistent with that found in many steady flows.

Key words: boundary layer stability

\section{Introduction}

Many industrial processes rely on the application of magnetic fields in order to control the stability of the system. Electromagnetic stirring, the casting of liquid metals and the growing of crystals (Organ \& Riley 1987; Hicks \& Riley 1989) are just a few examples where magnetic fields play a crucial role. In the case of the rotating-disk boundary layer, which occurs when a disk of infinite radius rotates beneath a stationary incompressible viscous body of fluid, several studies have shown that magnetic fields can greatly influence the instability characteristics. For a uniform magnetic field directed towards the disk surface, Jasmine \& Gajjar (2005) have concluded that a small magnetic field is all that is required to dramatically reduce the instability of the boundary layer.

Oscillatory fluid flows also occur in many processes and the paradigm example for investigating the disturbance characteristics of this class of flows is the flat Stokes layer. Its principal attraction lies in the fact that it is one of the few exact solutions to the unsteady Navier-Stokes equations and so many experimental and theoretical studies have been directed towards understanding its properties: a review of some of these is given by Davis (1976). More recently, studies have been carried out to assess the influence other effects may have on time-periodic flows. Gao \& Lu (2006a) proved that the imposition of suction is stabilizing, while it is also known that the presence of a surfactant can stabilize the Stokes layer as the critical Froude number is increased and the bandwidth of unstable modes shrinks (Gao \& Lu 2006b). It is in this spirit that here we make the first investigation of the effect of a magnetic field on the stability of the Stokes layer. 
A Stokes layer is generated when an infinitely long rigid plate oscillates sinusoidally in its own plane beneath a semi-infinite layer of incompressible viscous fluid that is at rest far away from the plate surface. It is characterized by two quantities as follows: the boundary-layer thickness $\delta \sim \sqrt{2 v / \omega}$ and the wall velocity $U_{0} \cos \omega t$, where $\omega$ is the frequency of the oscillating plate and $U_{0}$ is the maximum speed of the boundary. Combining these with the fluid viscosity, $v$, yields the Reynolds number

$$
R=\frac{U_{0} \delta}{2 v}=\frac{U_{0}}{\sqrt{2 v \omega}},
$$

which may be used to delineate the location for the onset of instability.

The linear stability of time-periodic flows may be determined using Floquet theory, which asserts that infinitesimal perturbations can be expressed in the form $\exp \left(\mu^{*} t\right) f$, where $f$ is a $2 \pi$-periodic function in time. The net growth or decay of a disturbance over a period is thereby subsumed in the real part of the Floquet exponent $\mu^{*}$; if this is positive then instability is present. The first successful application of Floquet theory to a fluid-stability problem was made by Seminara \& Hall (1976) who were concerned with the stability of a torsionally oscillating cylinder in an unbounded region of viscous fluid. When the technique was applied to the Stokes layer, Hall (1978) was unable to find any unstable modes for $R<160$ which was unexpected given the success of the method for the torsional cylinder flow. A related study by von Kerczek \& Davis (1974) considered the linear stability of the Stokes layer in a finite domain when a stationary lid was placed at a distance $8 \delta$ above the oscillating wall. They were able to take $R$ up to 400, but again could not detect any instability. This result is consistent with outcomes of some direct numerical simulations (DNSs) performed by Akhavan, Kamm \& Shapiro (1991) for $R \leqslant 500$.

These findings contradicted numerous experiments (Clamen \& Minton 1977; Eckmann \& Grotberg 1991) which show conclusively that in practice a flat Stokes layer certainly becomes unstable at sufficiently large $R$. The calculations mentioned above were limited by computational restrictions, but advancements in technology and numerical methods enabled Blennerhassett \& Bassom (2002) to extend the studies to much larger $R$. They were able to predict a critical Reynolds number for instability of $R_{c} \approx 708$ and traced out a portion of the neutral stability curve. Subsequent studies (Blennerhassett \& Bassom 2006, 2007) extended their investigation to oscillatory flow in channels and circular pipes. In these papers, the Floquet approach was abandoned in favour of pseudospectral methods based on the schemes outlined in Fornberg (1996) and Trefethen (2000). Reassuringly, at appropriately large channel widths the earlier results of Blennerhassett \& Bassom (2002) were retrieved and it was found that, in the case of the oscillating pipe, the critical $R$ could be lowered to about 564 for a pipe of optimal radius. Some of the predictions of the stability of the oscillatory flow in a channel have been confirmed by initial value calculations by Luo \& Wu (2010), while simulations carried out by Thomas et al. (2010) have verified the results of Blennerhassett \& Bassom (2002) for the semi-infinite Stokes layer.

In some flows (e.g. the Stokes layer), Floquet theory methods predict critical values of $R$ considerably larger than that observed in experiments. This discrepancy can occasionally be resolved by study of a quasi-steady limit in which the flow is thought of as frozen in time and an instantaneous growth rate of a disturbance calculated. This potentially can produce a transition mechanism at lower $R$ than predicted by Floquet theory. However, a rigorous justification of quasi-steady theory is often far from straightforward due to an underlying contradiction in the assumptions; on the one hand effectively freezing the basic flow requires $R \gg 1$, while the determination 
of a critical Reynolds number requires $R=O(1)$. Rather than discuss quasi-steady theory further here, we note that Hall (2003) used the technique to suggest that the Stokes layer is linearly stable in the $R \rightarrow \infty$ limit.

Here we apply both the Floquet and DNS methods to the unsteady flow that arises when a uniform axial magnetic field is directed towards a Stokes layer. In $\S 2$, the Floquet method is described and applied to the current magnetic field problem, while in $\S 3$ neutral curves and critical parameters for instability are presented. We conclude with a few remarks in $\S 4$.

\section{Formulation}

We consider a semi-infinite layer of electrically conducting viscous fluid confined by a flat plate located at $y=0$. The plate oscillates sinusoidally in its own plane along the $x$-direction with a velocity $U_{0} \cos \omega t$, while a uniform magnetic field $\boldsymbol{B}=B_{0} \boldsymbol{j}$ is applied to the system, where $\boldsymbol{j}$ is the unit vector in the $y$-direction. The dimensional Navier-Stokes equation that governs the fluid flow is given by

$$
\frac{\partial \boldsymbol{U}}{\partial t}+\boldsymbol{U} \cdot \nabla \boldsymbol{U}=-\frac{1}{\rho} \nabla P+\nu \nabla^{2} \boldsymbol{U}+\frac{1}{\rho} \boldsymbol{J} \times \boldsymbol{B},
$$

where the final term - the Lorentz force - takes into account the magnetic field effects. Denoting the electrical conductivity of the fluid by $\sigma$ and taking $\rho$ to be its density, the current density $\boldsymbol{J}=\sigma(\boldsymbol{E}+\boldsymbol{U} \times \boldsymbol{B})$ for an electric field $\boldsymbol{E}$ and fluid velocity $\boldsymbol{U}$. Coupling $(2.1 a)$ to

$$
\begin{gathered}
\frac{\partial \boldsymbol{B}}{\partial t}=\nabla \times(\boldsymbol{U} \times \boldsymbol{B})+\frac{1}{\sigma \mu_{0}} \nabla^{2} \boldsymbol{B}, \\
\nabla \cdot \boldsymbol{J}=0, \quad \nabla \times \boldsymbol{E}=-\frac{\partial \boldsymbol{B}}{\partial t}, \quad \nabla \cdot \boldsymbol{B}=0,
\end{gathered}
$$

leads to the full system of governing equations for the present magnetohydrodynamic problem; here $\mu_{0}$ specifies the magnetic permeability. The system is nondimensionalized by scaling all lengths upon the Stokes layer thickness, $\delta$, velocities on $U_{0}$ and putting $\tau=\omega t$. This throws up the magnetic Reynolds number $R_{m}=2 \sigma \mu_{0} \nu R$. In the subsequent modelling, the effects of the electric field are treated as negligible and it is supposed that $R_{m} \ll R$ so that the magnetic field is not affected by the motion of the fluid. This latter assumption is not particularly restrictive and holds true for many liquid metals. As a simple example, if we consider the case of mercury at room temperature, $\sigma=10^{6} \mathrm{~S} \mathrm{~m}^{-1}, \mu_{0}=4 \pi \times 10^{-7} \mathrm{~N} \mathrm{~A}^{-2}$ and $\nu=1.2 \times 10^{-7} \mathrm{~m}^{2} \mathrm{~s}^{-1}$ implying that the ratio of the fluid Reynolds number to the corresponding magnetic Reynolds number is $O\left(10^{7}\right)$.

Under these assumptions, $(2.1 a)$ yields the undisturbed basic dimensionless flow as simply

$$
\boldsymbol{U}=\left\{U_{B}(y, \tau), 0,0\right\}=\left\{\mathrm{e}^{-A y} \cos \left(\tau-\frac{y}{A}\right), 0,0\right\}
$$

where

$$
A^{2}=\sqrt{m^{2}+1}+m
$$

for a parameter $m \equiv B_{0}^{2} \sigma / \rho \omega$ that gives the appropriate measure of the strength of the applied magnetic field. 

form

The linear stability of the flow (2.2) is found by considering a disturbed flow of the

$$
\boldsymbol{U}=\left(U_{B}, 0,0\right)+\varepsilon\left(\frac{\partial \Psi}{\partial y},-\frac{\partial \Psi}{\partial x}, 0\right)
$$

where $\varepsilon \ll 1$ and $\Psi$ denotes the stream function of a two-dimensional disturbance. Squire's theorem has been successfully applied to non-conducting unsteady flows (von Kerczek \& Davis 1974), and it may easily be shown using standard methods that it can be extended to the current magnetic fluid flow. The upshot is that the above perturbation is sufficient for locating critical conditions for linear stability for the most unstable mode will be two-dimensional. The disturbance stream function, $\Psi$, is then written in exponential form

$$
\Psi=\mathrm{e}^{\mu \tau+\mathrm{i} a x} \psi(y, \tau)+\text { c.c. },
$$

where $a$ is the wavenumber, $\psi(y, \tau)$ is taken to be $2 \pi$-periodic in $\tau$ with any exponential growth or decay of $\Psi$ incorporated in the Floquet exponent $\mu$ and c.c. denotes the complex conjugate of the preceding expression. The corresponding vorticity perturbation is then expressed as $\mathrm{e}^{\mu \tau+\mathrm{i} a x} \zeta(y, \tau)+$ c.c., whereupon the governing stream function-vorticity equations, when linearized in $\varepsilon$, may be reduced to

$$
\begin{aligned}
\frac{\partial \zeta}{\partial \tau} & =\frac{1}{2}\left(\frac{\partial^{2}}{\partial y^{2}}-a^{2}\right) \zeta-\mu \zeta-\mathrm{i} a R U_{B} \zeta-m \frac{\partial^{2}}{\partial y^{2}} \psi+\mathrm{i} a R U_{B}^{\prime \prime} \psi, \\
\zeta & =\left(\frac{\partial^{2}}{\partial y^{2}}-a^{2}\right) \psi .
\end{aligned}
$$

This system needs to be solved subject to the boundary conditions

$$
\psi=\psi^{\prime}=0 \quad \text { on } y=0 ; \quad \psi, \psi^{\prime} \rightarrow 0 \quad \text { as } y \rightarrow \infty,
$$

where a prime denotes differentiation with respect to $y$. In this formulation, $a$ is real-valued, while the eigenvalue $\mu$ is complex although its imaginary part $\mu_{i}$ can be restricted to the range $0 \leqslant \mu_{i} \leqslant 0.5$ (Blennerhassett \& Bassom 2002).

The unknown stream function is decomposed into harmonics

$$
\psi=\sum_{n=-\infty}^{n=\infty} \psi_{n}(y) \exp \{\mathrm{in} \tau\},
$$

so that equating coefficients of harmonics in $(2.6 a, b)$ generates the infinite system of ordinary differential equations

$$
\left[\mathscr{L}^{2}-2\{\mu+\mathrm{i} n\} \mathscr{L}-2 m \mathscr{D}^{2}\right] \psi_{n}=\mathrm{i} a R\left[\mathrm{e}^{-(A+\mathrm{i} / A) y} \mathscr{M}_{-} \psi_{n-1}+\mathrm{e}^{-(A-\mathrm{i} / A) y} \mathscr{M}_{+} \psi_{n+1}\right],
$$

where

$$
\mathscr{L} \equiv \frac{\partial^{2}}{\partial y^{2}}-a^{2}, \quad \mathscr{D}^{2} \equiv \frac{\partial^{2}}{\partial y^{2}} \quad \text { and } \quad \mathscr{M}_{ \pm} \equiv \mathscr{L}-A^{2}+A^{-2} \pm 2 \mathrm{i} .
$$

The system (2.8) was solved numerically using the pseudospectral techniques described by Fornberg (1996) and Trefethen (2000). Such methods were detailed in Blennerhassett \& Bassom (2006) so we only provide very brief details of the numerical techniques involved here. The differential operators appearing in (2.8) were replaced by their respective pseudospectral matrix approximations and each $\psi_{n}(y)$ 
cast as a vector $\psi_{n}$ of its function values on a Chebyshev mesh. We introduce the matrix operators

$$
\begin{aligned}
\mathscr{L} & \rightarrow \boldsymbol{L}, \quad \mathscr{D}^{2} \rightarrow \boldsymbol{D}^{2}, \quad \frac{1}{2}\left(\frac{\partial^{4}}{\partial y^{4}}-2 a^{2} \frac{\partial^{2}}{\partial y^{2}}+a^{4}\right) \rightarrow \boldsymbol{V}, \\
\boldsymbol{M} & =\frac{1}{2} \boldsymbol{L}^{-1} \mathrm{e}^{-(A+\mathrm{i} / A) y}\left(\boldsymbol{L}-\left\{\left[A^{2}-A^{-2}\right]+2 \mathrm{i}\right\} \boldsymbol{I}\right),
\end{aligned}
$$

which allow us to rearrange the general governing (2.8) as

$$
-\mathrm{i} a R \widetilde{\boldsymbol{M}} \boldsymbol{\psi}_{n+1}+\left(\boldsymbol{L}^{-1}\left\{\boldsymbol{V}-m \boldsymbol{D}^{2}\right\}-\mathrm{i} n \boldsymbol{I}\right) \boldsymbol{\psi}_{n}-\mathrm{i} a R \boldsymbol{M} \boldsymbol{\psi}_{n-1}=\mu \boldsymbol{\psi}_{n},
$$

where $\boldsymbol{I}$ is the identity matrix and $\widetilde{\boldsymbol{M}}$ is the complex conjugate of $\boldsymbol{M}$. A finite system of equations is obtained by truncating the Fourier series (2.7) for $\psi$ and setting $\psi_{n} \equiv 0$ for all $|n|>N$. The system (2.11) can now be written as the algebraic eigenvalue problem

$$
\boldsymbol{A} \boldsymbol{\phi}=\mu \boldsymbol{\phi},
$$

where $\boldsymbol{A}$ is a sparse matrix and the vector $\boldsymbol{\phi}$ is given by

$$
\boldsymbol{\phi}^{\mathrm{T}}=\left(\boldsymbol{\psi}_{N}^{\mathrm{T}} \boldsymbol{\psi}_{N-1}^{\mathrm{T}}, \ldots, \boldsymbol{\psi}_{0}^{\mathrm{T}}, \ldots, \boldsymbol{\psi}_{-N}^{T}\right) \text {. }
$$

\section{Results}

Solutions to the eigenvalue problem (2.12) were obtained using the sparse-matrix eigenvalue routines that are available in Matlab. Several checks were conducted to ensure that the computed eigenvalues and eigenvectors possessed at least five decimal place accuracy. Of critical importance is the determination of the value $N$ that governs the point at which the Fourier series (2.7) is truncated. If $N$ is too small the computed values are inaccurate, but if it is too large then the calculations are lengthy and inefficient. It was found empirically that the choice $N \approx 0.8 a R$ works well, while at least $K=50$ data points were required in the $y$-direction to ensure satisfactory behaviour. If either $N$ or $K$ were less than these values, the predicted growth rate tended to be over-estimated and so gave a misleading representation of the disturbance behaviour. Figure 1 displays the neutral stability curves in the wavenumber-Reynolds number $(a, R)$-plane for several magnetic parameters within the range $m \in[0,0.2]$. The solid lines illustrate the results obtained using the Floquet eigenvalue method; values were accurately computed at neutral points $\left(a_{j}, R\left(a_{j}\right)\right)$ separated by a wavenumber increment $\Delta a_{j}=0.01$ and then interpolation used to infer the remainder of the curve. It is apparent that a magnetic field is stabilizing; furthermore only a modest field is necessary to have a significant effect, since as $m$ is increased from 0 to 0.2 , the corresponding critical Reynolds number more than doubles. In the context of mercury at room temperature, forced to oscillate at $1 \mathrm{~Hz}$, we have $\rho=1.35 \times 10^{4} \mathrm{~kg} \mathrm{~m}^{-1}$ and then a magnetic field strength $B_{0}=0.052 \mathrm{~T}$ would be required to achieve the stabilizing effects observed for $m=0.2$.

We remark in passing that the smoothed neutral curves in figure 1 are a slight simplification of the actual curves. In their study of the non-conducting Stokes layer, Blennerhassett \& Bassom (2002) showed that strictly the neutral curves are punctuated by small spikes which represent disturbances that are stationary relative to the underlying basic flow (i.e. $\mu=0$ ). In many regards, these spikes are incidental to the overall structure of the neutral curves for they protrude a distance less than unity in Reynolds number space. These spikes also exist in our current problem, but the 


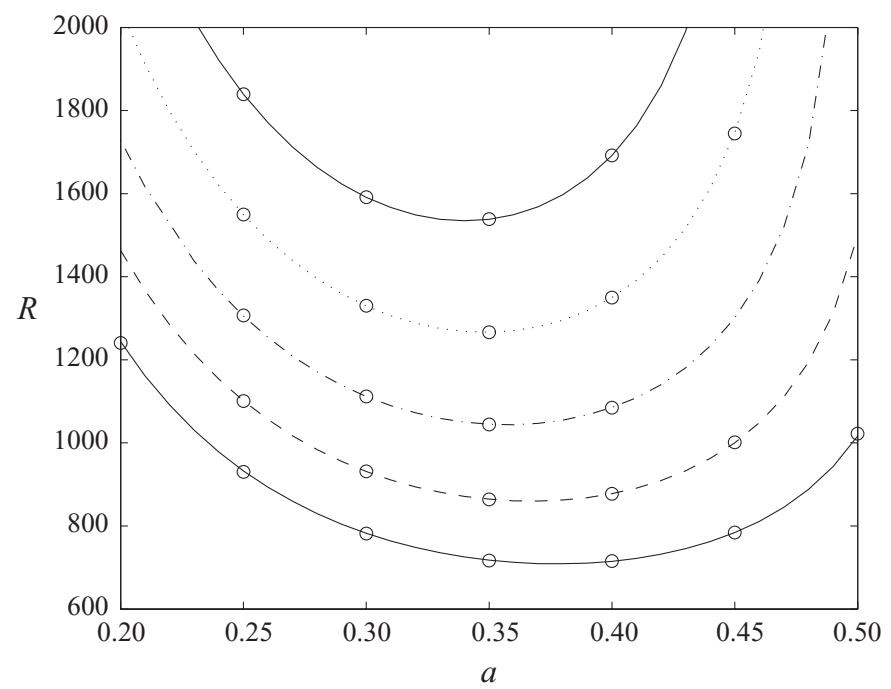

FIGURE 1. Neutral stability curves for $m=0,0.05,0.1,0.15$ and 0.2 . The lowest curve corresponds to the zero magnetic field and increases in $m$ move the curves upwards in the obvious manner. The lines mark the results obtained using Floquet theory, while points indicated with $\bigcirc$ show corresponding DNS predictions.

resulting changes in critical $R$ and wavenumber would be insignificant and certainly not visible on the scale of figure 1.

As a check on our results a velocity-vorticity formulation of the fully linearized Navier-Stokes equations was employed. This scheme is based on the methods described by Davies \& Carpenter (2001) and uses Chebyshev discretization in the normal-to-wall direction together with a Fourier spectral scheme in the $x$-direction and combined with semi-implicit methods for the time-stepping procedure. Time histories of the disturbance evolution are obtained that allow the growth or decay of a perturbation to be calculated, which in turn is used to determine critical stability. A detailed description of the DNS implementation may be found in Thomas et al. (2010).

Selected points on the stability curves in figure 1 were re-calculated using these DNS methods and the results are denoted by the $\bigcirc$ symbols in figure 1. A timedependent impulsive forcing was used to initiate perturbations containing a spectrum of frequencies and then that with the greatest growth rate evolved and dominated the remainder. Accurate results were obtained by implementing time steps $\Delta \tau=0.1 / R$ and using 100 Chebyshev polynomials, while the net growth or decay rate, $\mu_{r}$, of a disturbance was estimated by calculating $\ln |\zeta(\tau+2 \pi) / \zeta(\tau)| / 2 \pi$, where $\zeta$ is the characteristic size of the vorticity perturbation on the wall. Critical points along the neutral stability curve were then estimated by linear interpolation on a pair of closely spaced points $\left(\mu_{r}^{ \pm}, R^{ \pm}(a)\right)$ for a fixed wavenumber $a$ and $\mu_{r}^{ \pm}<0.02$. The DNS results are plotted at wavenumber intervals $\Delta a=0.05$ and these predictions are seen to be in a very good agreement with those obtained using the Matlab methods. In passing, we remark that although negative values of the magnetic parameter $m$ can be precluded on physical grounds, since they imply a negative electrical conductivity, they can be used to provide further validation of the two numerical methods. The Floquet and DNS results agree in predicting that if $m<0$ there would be a destabilization of the flow at lower Reynolds numbers. 
(a)

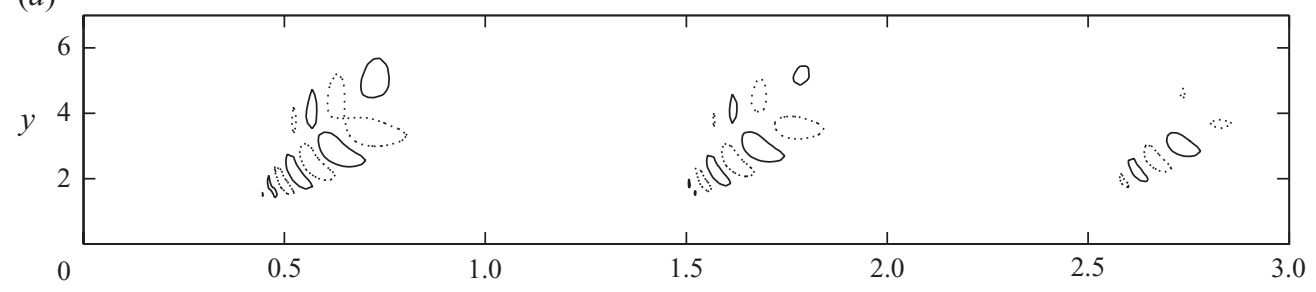

(b)
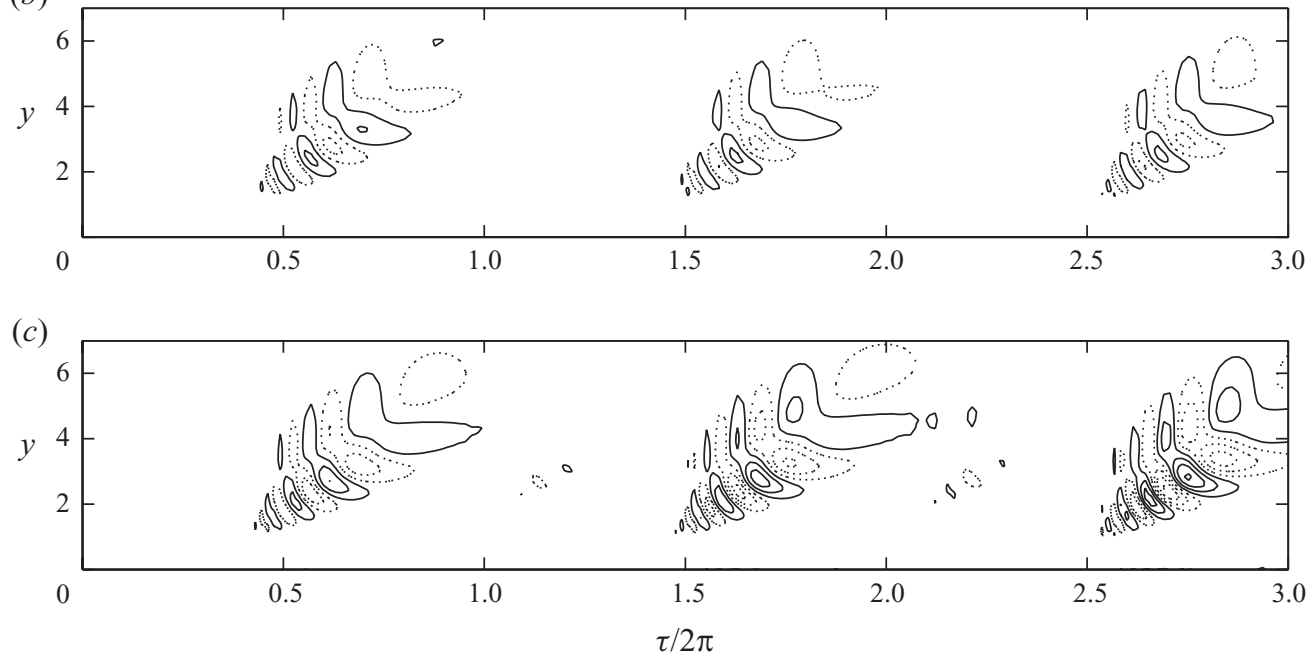

FIGURE 2. Contours of the vorticity perturbation, $\zeta$, taken over three cycles of oscillation with magnetic parameter $m=0.1$, wavenumber $a=0.4$ and $(a) R=1075$, (b) $R=1085$ and (c) $R=1095$. Positive contours are represented by solid lines, while negative contours are indicated by dotted lines.

Vorticity perturbation contours in $(y, \tau / 2 \pi)$ space obtained via DNS, are displayed in figure 2. The disturbance evolution is plotted over three periods for the magnetic field $m=0.1$, a wavenumber $a=0.4$ and the three Reynolds numbers $R=1075,1085$ and 1095. The three simulations display similar features: there is one source of vorticity per cycle, which originates near the plate surface and develops along the $y$-axis, eventually decaying at a sufficiently far distance from the wall. However, as the Reynolds numbers differ, so the magnitude of the vorticity varies as time advances. When $R=1075$, temporal net decay is observed, as the vorticity shrinks from one cycle to the next, whilst net growth is displayed for $R=1095$. The eye cannot detect net growth or decay at the intermediate $R=1085$, which is consistent with the neutral results of figure 1 that this choice is very close to critical instability. Also it is of interest that there is strong amplification of vorticity into regions where the shear stress decays to zero. On the face of it, this is somewhat surprising, but such behaviour has been observed by Healey (2006) for the steady rotating-disk boundary layer; furthermore he suggested a mechanism by which sustained growth could occur in a region with no Reynolds stress.

The strongly stabilizing effect of an axial magnetic field is further emphasized in figure 3. Here the solid line illustrates the behaviour of the critical Reynolds number against $m$, while the dashed curve displays the change in the critical wavenumber. For $m=0.3, R_{c} \approx 2236$, which is more than three times that of the case corresponding 


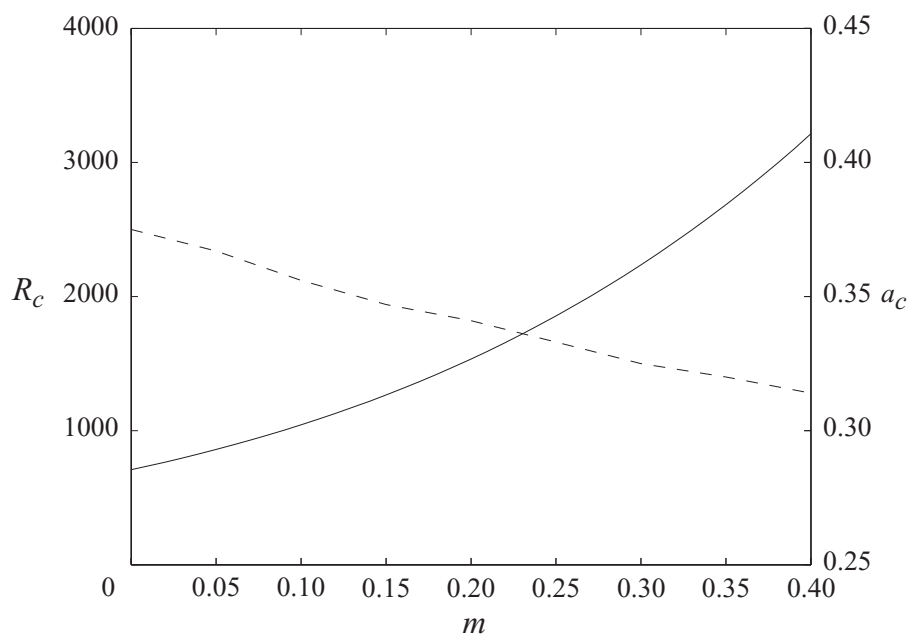

FIGURE 3. Critical results for the Reynolds number (solid line) and wavenumber (dashed line) as a function of the magnetic field parameter, $m$.

to a zero magnetic field. The figure also illustrates the less pronounced effect on the critical wavenumber, which reduces as $m$ is increased.

\section{Final remarks}

In this study, we have endeavoured to characterize the effects resulting from the imposition of an axial magnetic field on the classical flat Stokes layer. The dramatic stabilization induced by the magnetic field has been confirmed using both Floquet theory methods implemented using pseudospectral schemes and the DNS strategy developed by Davies \& Carpenter (2001). The neutral curves and critical parameters for instability prove that an axial magnetic field is stabilizing. As with the rotatingdisk boundary layer, the magnetic field has the effect of reducing the boundary-layer thickness, and thus only small increments in the magnetic parameter, $m$, are required to greatly alter the stability characteristics of the Stokes layer. Hence, the effect is qualitatively similar to that due to mass suction, which may also be used to suppress instability (Gao \& Lu 2006a).

This work was supported by the Australian Research Council grant DP0880463. We are grateful to the referees for several suggestions which have led to a much improved paper.

\section{REFERENCES}

Akhavan, R., Kamm, R. D. \& Shapiro, A. H. 1991 An investigation of transition to turbulence in bounded oscillatory Stokes flows. Part 2. Numerical simulations. J. Fluid Mech. 225, 423-444.

Blennerhassett, P. J. \& Bassom, A. P. 2002 The linear stability of flat Stokes layers. J. Fluid Mech. 464, 393-410.

Blennerhassett, P. J. \& Bassom, A. P. 2006 The linear stability of high-frequency oscillatory flow in a channel. J. Fluid Mech. 556, 1-25.

Blennerhassett, P. J. \& Bassom, A. P. 2007 The linear stability of high-frequency flow in a torsionally oscillating cylinder. J. Fluid Mech. 576, 491-505.

Clamen, M. \& Minton, P. 1977 An experimental investigation of flow in an oscillatory pipe. J. Fluid Mech. 77, 421-431. 
Davies, C. \& CARPenter, P. W. 2001 A novel velocity-vorticity formulation of the Navier-Stokes equations with applications to boundary layer disturbance evolution. J. Comp. Phys. 172, $119-165$.

Davis, S. H. 1976 The stability of time-periodic flows. Annu. Rev. Fluid Mech. 8, 57-74.

Eckmann, D. M. \& Grotberg, J. B. 1991 Experiments on transition to turbulence in oscillatory pipe flow. J. Fluid Mech. 222, 329-350.

Fornberg, B. 1996 A Practical Guide to Pseudospectral Methods. Cambridge University Press.

GAO, P. \& LU, X. Y. $2006 a$ Effect of wall suction/blowing on the linear stability of flat Stokes layers. J. Fluid Mech. 551, 303-308.

GaO, P. \& LU, X. Y. $2006 b$ Effect of surfactants on the long-wave stability of oscillatory fluid flow. J. Fluid Mech. 562, 345-354.

Hall, P. 1978 The linear stability of flat Stokes layers. Proc. R. Soc. Lond. A 359, 151-166.

HaLl, P. 2003 On the stability of the Stokes layers at high Reynolds numbers. J. Fluid Mech. 482, $1-15$.

Healey, J. J. 2006 A new convective instability of the rotating-disk boundary layer with growth normal to the disk. J. Fluid Mech. 560, 279-310.

Hicks, T. W. \& RiLey, N. 1989 Boundary layers in magnetic Czochralski crystal growth. J. Cryst. Growth 96, 957-968.

JASMine, H. A. \& GAJJAR, J. S. B. 2005 Convective and absolute instability in the incompressible boundary layer on a rotating disk in the presence of a uniform magnetic field. J. Engng Maths 52, 337-353.

von KerczeK, C. \& Davis, S. H. 1974 Linear stability theory of oscillatory Stokes layers. J. Fluid Mech. 62, 753-773.

LuO, J. \& WU, X. 2010 On the linear stability of a finite Stokes layer: instantaneous versus Floquet modes. Phys. Fluids 22, 054106.

Organ, A. E. \& Riley, N. 1987 Oxygen transport in magnetic Czochralski growth of Silicon. J. Cryst. Growth 82, 465-476.

Seminara, G. \& Hall, P. 1976 Centrifugal instability of a Stokes layer. Proc. R. Soc. Lond. A 350, 299-316.

Thomas, C., Bassom, A. P., Blennerhassett, P. J. \& Davies, C. 2010 Direct numerical simulations of small disturbances in the classical Stokes layer. J. Engng Maths, doi: 10.1007/S10665-0109381-0.

Trefethen, L. N. 2000 Spectral Methods in MATLAB. SIAM. 